Research Paper

\title{
High serum haptoglobin level is associated with tumor progression and predicts poor prognosis in non-small cell lung cancer
}

\author{
Jianjun Lu1,2,*, Yanhong Wang ${ }^{3, *}$, Miansheng Yan ${ }^{1, *}$, Pinning Feng ${ }^{1}$, Linjing Yuan ${ }^{4}$, \\ Yuesu Cai ${ }^{1,5}$, Xin Xia ${ }^{2}$, Min Liu ${ }^{1}$, Jinmei Luo ${ }^{1,3}$, Laisheng $\mathrm{Li}^{1}$ \\ ${ }^{1}$ Department of Laboratory Medicine, The First Affiliated Hospital of Sun Yat-Sen University, Guangzhou, 510080, People's \\ Republic of China \\ ${ }^{2}$ Department of Thoracic Surgery, The First Affiliated Hospital of Sun Yat-Sen University, Guangzhou, 510080, People's \\ Republic of China \\ ${ }^{3}$ Department of Internal Medicine, Medical Intensive Care Unit and Division of Respiratory Diseases, Third Affiliated Hospital \\ of Sun Yat-Sen University, Guangzhou, 510630, People's Republic of China \\ ${ }^{4}$ Department of Gynaecology, The First Affiliated Hospital of Sun Yat-Sen University, Guangzhou, 510080, People's Republic \\ of China \\ ${ }^{5}$ Institute of Laboratory Medicine, Guangdong Medical University, Dongguan, 523808, People's Republic of China \\ *These authors contributed equally to this work \\ Correspondence to: Laisheng Li, email: lilaish@mail.sysu.edu.cn \\ Jinmei Luo, email: luojm3@mail.sysu.edu.cn
}

Keywords: haptoglobin, NSCLC, progression, prognosis, biomarker

Received: April 26, 2016

Accepted: May 22, 2016

Published: May 27, 2016

\section{ABSTRACT}

The overall survival time of non-small cell lung cancer (NSCLC) has not improved dramatically in recent decades. An important reason is the lacking of valuable biomarkers. Haptoglobin was reported to have activities of anti-inflammatory, antioxidant, autoimmune and tumor angiogenesis. However its potential role as a tumor biomarker was not well recognized. We used an immunoturbidimetry method to measure serum haptoglobin levels in 205 NSCLC patients, and 210 normal healthy controls. We found that serum haptoglobin levels were significantly elevated in NSCLC patients compared with normal healthy controls $(1.985 \pm 1.039 \mathrm{mg} / \mathrm{mLvs} .0 .922 \pm$ $0.495 \mathrm{mg} / \mathrm{mL}$, respectively, $P<0.0001$ ). Higher serum haptoglobin levels were associated with advanced TNM stage, lymph node metastasis, and distant metastasis. Area under receiver operating characteristic curve (ROC) for serum haptoglobin was 0.809 (95\% CI: $0.767-0.852)$ at a specificity of 0.881 and sensitivity of 0.639 . The optimal cut-off value of haptoglobin was $1.495 \mathrm{mg} / \mathrm{mL}$ for discriminating NSCLC from normal healthy controls. Kaplan-Meier log rank analysis revealed that the higher serum haptoglobin levels group had a poorer overall survival compared with lower haptoglobin group (the median survival was 12.0 weeks, 26.0 weeks, respectively, $P<0.01)$. Further univariate and multivariate Cox regression analysis showed that serum haptoglobin was an independent risk factor of prognosis of NSCLC patients ( $P<0.01, P=0.01$, respectively). In conclusion, our study suggests that serum haptoglobin may act as useful clinical serological biomarkers in progression and prognostic evaluation in NSCLC.

\section{INTRODUCTION}

Lung cancer remains the most frequent incident cancer and the premier cause of cancer-related mortality for both men and women in China [1]. The most commonly diagnosed lung cancers are non-small cell lung cancer (NSCLC), which accounts for up to $85 \%$. There are three histological subtypes of NSCLC, adenocarcinoma, squamous cell carcinoma, and large cell carcinoma [2]. NSCLC prognosis is deeply hinge 
on early stage diagnosis, more than $50 \%$ of patients with early stage diseases survive more than 5 years, compared with 5 year survival rate of less than $5 \%$ in patients with advanced stage diseases [3]. These lead many physicians and investigators to hunt for early screening strategies, including novel biomarkers and diagnostic tools to detect early stage NSCLC. However, those examination methods including imaging with chest X-rays, low dose computed tomography scans [4], sputum exfoliative cytological analysis, and a series of serum biomarker, such as CEA, NSE, CYFRA21-1, and SCC [5], have little to do with displaying the mortality of lung cancer. Therefore, investigation of a novel biomarker is clinically urgently needed.

Haptoglobin, an acute phase reactant protein, mainly produced by liver but also by other tissues, such as lung, spleen, kidney, skin and adipose tissue [6], which is constituted by an alpha chain and a beta chain, displaying in humans as three major phenotypes, Hp1-1, Hp2-2 and Hp2-1 [7]. It mainly functioned in the area of anti-inflammatory and anti-oxidant, but also in the field of autoimmune diseases, neurodegenerative diseases, and tumor angiogenesis [8, 9]. Elevated levels of serum haptoglobin were found in patients with inflammatory diseases [10] and a variety of cancers, including lung cancer [11-14]. We supposed that the serum levels of haptoglobin might be a good biomarker for progression and prognosis of NSCLC patients. However, to date, there is no report about the association between serum haptoglobin levels and prognosis of NSCLC.

In this study, to investigate the application of serum haptoglobin as a diagnostic potential biomarker for NSCLC, we measured the levels of serum haptoglobin in NSCLC patients by turbidimetric immunoassay and validated their ability to predict prognosis in NSCLC.

\section{RESULTS}

\section{Clinical characteristics of patients}

In this study, we recruited a total of 205 NSCLC patients and 210 normal healthy controls. The demographic, pathologic, and clinical information of the study subjects were displayed in Table 1 . The mean age of the NSCLC patients (61.6, years) was not obviously different from normal healthy controls (60.6, years). The proportion of male gender accounted for $65.4 \%$ of the NSCLC patients and $57.1 \%$ of the normal healthy controls, respectively, with no significantly difference. However, the proportion of smoking status was apparently more in NSCLC patients than normal healthy controls, which was $71.7 \%$ vs. $56.2 \%$. More than half of the patients were in stage III + IV $(70.2 \%), 63.4 \%$ of the patients with lymph node metastases, and $24.4 \%$ with distant metastases.

\section{Association between serum haptoglobin levels and clinicopathological variables}

To determine the potential of haptoglobin as a serological biomarker for NSCLC, we analyzed the levels of serum haptoglobin in NSCLC patients and normal healthy controls by turbidimetric immunoassay. The mean serum haptoglobin level in the NSCLC patients $(1.985 \pm 1.039 \mathrm{mg} / \mathrm{mL})$ was significantly elevated, compared with the normal healthy control group $(0.922 \pm 0.495 \mathrm{mg} / \mathrm{mL})(P<0.0001$, Figure $1 \mathrm{~A})$. The statistical power value was $1.0(>0.8$ was considered significant). We further evaluated the clinicopathologic significance of the serum haptoglobin level in NSCLC patients. Table 2 summarized the association between the serum haptoglobin levels and clinicopathological variables in NSCLC patients. As displayed in Figure 1B, when compared with the normal healthy controls, the serum haptoglobin were notably elevated in NSCLC patients at both early TNM stage (stage I + II) and advanced TNM stage (stage III + IV), and serum haptoglobin level was even higher in advanced stage patients than early stage patients. Furthermore, the serum haptoglobin levels were obviously higher in patients with lymph node metastases than those without $(2.136 \pm 1.077 \mathrm{mg} / \mathrm{mL}, 1.719 \pm 0.917$ $\mathrm{mg} / \mathrm{mL}$, respectively, $P=0.0356$ ) (Table 2, Figure 1C). Meanwhile, statistically significant differences in haptoglobin levels were found between NSCLC patients with distant metastases and those patients without distant metastases $(2.354 \pm 1.069 \mathrm{mg} / \mathrm{mL}, 1.867 \pm 1.005 \mathrm{mg} / \mathrm{mL}$, respectively, $P=0.0004$ ) (Table 2 , Figure 1D). Besides, the serum haptoglobin levels were observed have no significant differences from other clinicopathological variables (Table 2). After all, these results indicated that serum haptoglobin levels increased in NSCLC patients, and associated with the progression and metastasis NSCLC, which could be serve as a potential biomarker to differentiate NSCLC patients from healthy controls, even the indicator for prognosis.

\section{ROC analysis of serum haptoglobin levels in NSCLC patients}

To evaluate the value of serum haptoglobin as a biomarker for NSCLC diagnosis, we calculated the ROC/AUC through plotting sensitivity against specificity at different sort for serum haptoglobin. At the beginning, we assess the value of serum haptoglobin for discriminating NSCLC patients from normal healthy controls, ROC/AUC analysis displayed a sensitivity of 0.639 (specificity of $0.881, \mathrm{AUC}=0.809,95 \% \mathrm{CI}$ : $0.767-0.852$, cut-off value $=1.495 \mathrm{mg} / \mathrm{mL}$, Figure $2 \mathrm{~A}$ ). Then, in order to discriminate NSCLC patients without lymph node metastases from normal healthy controls, ROC/AUC analysis reached a sensitivity of 0.568 
Table 1: Clinicopathological variables of NSCLC patients and normal healthy controls $(n, \%)$

\begin{tabular}{lcc}
\hline \multicolumn{1}{c}{ Variables } & NSCLC cases $(\boldsymbol{n}=\mathbf{2 0 5})$ & Normal controls $(\boldsymbol{n}=\mathbf{2 1 0})$ \\
\hline Age (years) & $61.6 \pm 9.8$ & $60.6 \pm 11.1$ \\
Gender $(n, \%)$ & $134(65.4)$ & $120(57.1)$ \\
Male & $71(34.6)$ & $90(42.9)$ \\
Female & & $92(43.8)$ \\
Smoking status $(n, \%)$ & $58(28.3)$ & $118(56.2)$ \\
Non-smoker & $147(71.7)$ & \\
Smoker & & \\
Histology & $116(56.6)$ & \\
Adenocarcinoma & $76(37.1)$ & \\
Squamous cell carcinoma & $13(6.3)$ & \\
Other & & \\
TNM stage & $61(29.8)$ & \\
I+II & $144(70.2)$ & \\
III+IV & & \\
Differentiation & $156(22.4)$ & \\
Well & $144(70.3)$ & \\
Moderate and low & $15(7.3)$ & \\
Unknown & & \\
Lymph node metastases & & \\
Negative & & \\
Positive & & \\
Distant metastases & & \\
Negative & & \\
Positive & & \\
\hline
\end{tabular}

(specificity of $0.843, \mathrm{AUC}=0.762,95 \% \mathrm{CI}: 0.692-0.832$, cut-off value $=1.405 \mathrm{mg} / \mathrm{mL}$, Figure $2 \mathrm{~B}$ ). We further to distinguish NSCLC patients with lymph node metastases from normal healthy controls, ROC/AUC analysis showed a sensitivity of 0.664 (specificity of $0.881, \mathrm{AUC}=0.836$, $95 \%$ CI: $0.788-0.884$, cut-off value $=1.495 \mathrm{mg} / \mathrm{mL}$, Figure 2C). Last, to predict NSCLC patients lymph node metastasis status, we compare the NSCLC patients with lymph node metastasis or not, data presented a sensitivity of 0.672 (specificity of $0.554, \mathrm{AUC}=0.609,95 \% \mathrm{CI}$ : $0.530-0.688$, cut-off value $=1.685 \mathrm{mg} / \mathrm{mL}$, Figure 2D). After all, we use serum haptoglobin levels at $1.495 \mathrm{mg} / \mathrm{mL}$ as cut-off value of NSCLC for subsequently analysis.

\section{Serum haptoglobin levels are an independent prognostic indicator for overall survival of NSCLC patients}

To evaluate the prognostic significance of the serum haptoglobin levels, we used serum haptoglobin cut-off value $1.495 \mathrm{mg} / \mathrm{mL}$, which was calculated from previous ROC analysis, as a threshold to partitioned 205 NSCLC patients into two groups, high serum haptoglobin group (haptoglobin $\geq 1.495 \mathrm{mg} / \mathrm{mL}, n=131$ ) and low serum haptoglobin group (haptoglobin $<1.495 \mathrm{mg} / \mathrm{mL}, n=74$ ). Overall survival time was calculated from the date of sampling to the date of death from cancer (cancerspecific survival). Deaths caused from not cancer-related, unknown factors, and subjects alive were censored. As shown by Kaplan-Meier log rank analysis, the higher serum haptoglobin levels group was correlated with a poorer overall survival, compared with lower serum haptoglobin levels group, the median survival time were 12.0 weeks (95\% CI, 10.3 to 13.7$)$ and 26.0 weeks (95\% CI, 20.3 to 31.7$)$, respectively, $(P<0.01$, Figure 3$)$. Further analysis using univariate and multivariate Cox regression showed that TNM stage, lymph node metastasis status, pathological distant metastasis status, and serum haptoglobin levels were independent risk factors of prognosis of NSCLC patients (Table 3). 


\section{DISCUSSION}

In the present study, the diagnostic and prognosis value of serum haptoglobin levels in NSCLC patients were determined and evaluated. We found that serum haptoglobin levels were obviously elevated in NSCLC patients compared with controls. Unfavorable clinicopathological variables, TNM stage, lymph node metastasis, and distant metastasis, were associated with high serum haptoglobin levels. Kaplan-Meier and Cox regression analysis revealed that high haptoglobin levels were correlated with poor overall survival and it could become an independent prognostic factor for NSCLC. Furthermore, ROC analysis displayed that serum haptoglobin level had a potential to distinguish NSCLC patients from normal healthy controls. Considering the haptoglobin test can be easily performed in the conventional clinical biochemistry laboratory by commercially available kit, our results strongly suggest that serum haptoglobin may serve as a useful serum biomarker for the diagnosis and prognosis of NSCLC.

Blood sample is convenient to collection, and the test data can be stable replication during long periods, serological biomarkers have been regarged as valuable indicator in the diagnosis, treatment and prognosis of lung cancer [15]. Accumulating studies in serum sample displayed the pros and cons of usual serum biomarker for NSCLC diagnosis [16-18], while others emphasized their function in disease progression, therapy and prognosis evaluation [19]. Yang $D$ et al. measured cytokine contrations by using multiplexed cytokine immunoassays, and found an NSCLC-specific profile of inflammatory mediators, including CXCL10, CXCL11 and CCL20 which would be useful for predicting of therapeutic effects and overall survival [20]. Wikoff WR et al. applied a liquid chromatography/mass spectrometry hydrophilic interaction method to analyze a wide rage of serum metabolites, and identified diacetylspermine was a novel serum metabolite with obvious performance in prediagnostic NSCLC [21]. From the clinical investigator's standpoint, CA125, CEA, CYFRA21-1 and SCC still play as the leading serum biomarker in NSCLC and are mainly applied in disease monitoring[17]. Therefore, novel biomarker for diagnosis and prognosis evaluation would assist the doctors to improve the clinical management of NSCLC. The present study was performed to evaluate serum haptoglobin value of diagnostic and prognostic in NSCLC.

In NSCLC, Hoagland $L F$ et al. found serum haptoglobin levels significantly elevated in NSCLC patients compared with normal controls, and strongly associated with NSCLC stage [11]. However, this study's subject quantity is not good enough, and no prognostic data. Park J et al. utilized stable isotope dilution-multiple
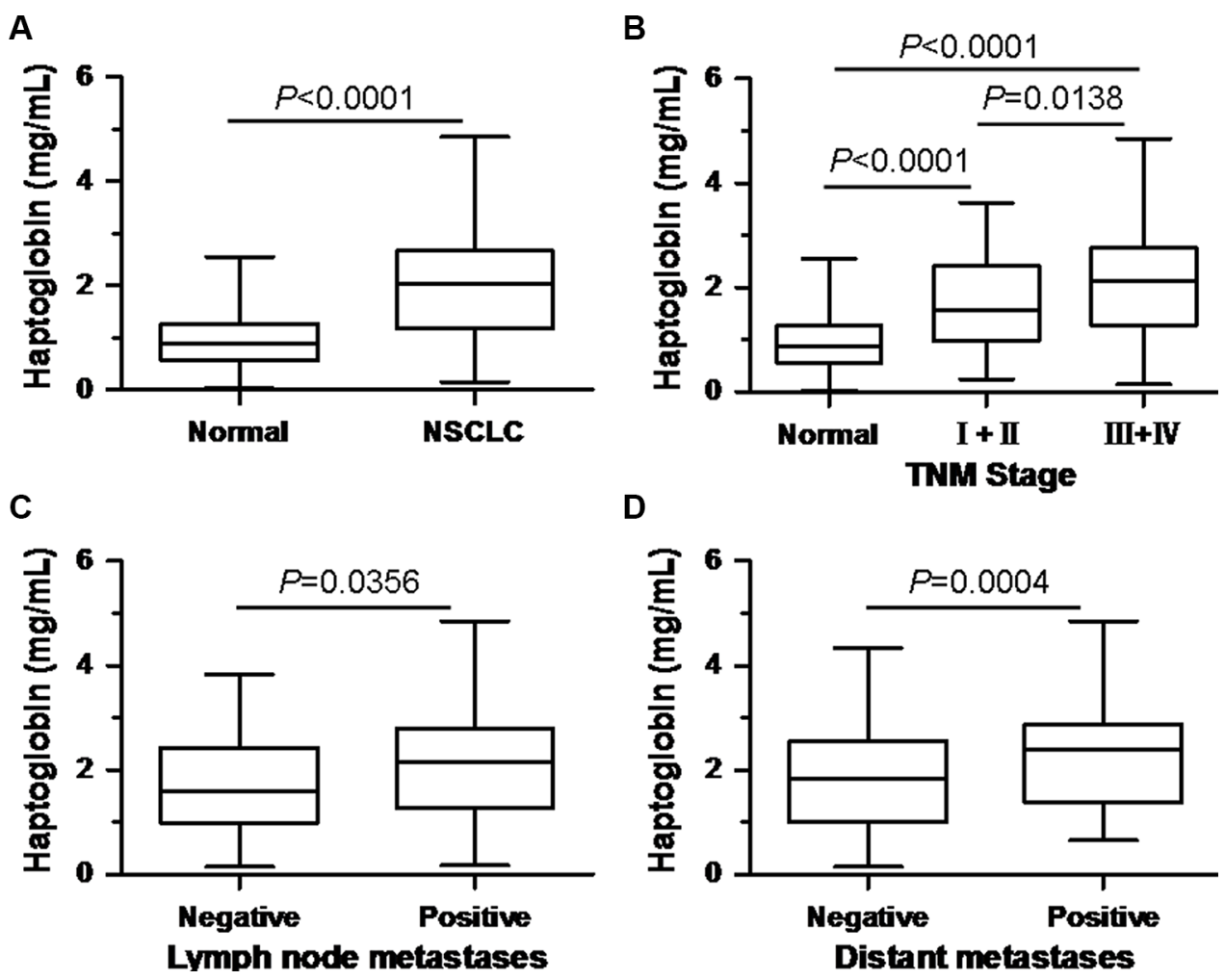

Figure 1: Comparison of serum haptoglobin levels (A) between normal healthy controls and NSCLC patients; (B) in normal healthy controls and NSCLC patients at different TNM stage; (C) in NSCLC patients with and without lymph node metastasis; (D) in NSCLC patients with and without distant metastasis. 
Table 2: Association between serum Haptoglobin levels and characteristical variables in NSCLC patients

\begin{tabular}{|c|c|c|c|}
\hline Variables & Number & Haptoglobin $(\mathrm{mg} / \mathrm{mL})$ & $P$ values \\
\hline \multicolumn{4}{|l|}{ Age $(Y)$} \\
\hline$\leq 60$ & 121 & $1.976 \pm 1.098$ & 0.9240 \\
\hline$>60$ & 84 & $1.996 \pm 0.972$ & \\
\hline \multicolumn{4}{|l|}{ Gender } \\
\hline Male & 134 & $1.971 \pm 1.029$ & 0.6071 \\
\hline Female & 71 & $2.014 \pm 1.066$ & \\
\hline \multicolumn{4}{|l|}{ Smoking status } \\
\hline Non-smoker & 58 & $1.889 \pm 0.920$ & 0.6772 \\
\hline Smoker & 147 & $2.023 \pm 1.084$ & \\
\hline \multicolumn{4}{|l|}{ Histology } \\
\hline Adenocarcinoma & 116 & $2.009 \pm 1.023$ & 0.8547 \\
\hline Squamous cell carcinoma & 76 & $1.964 \pm 1.045$ & \\
\hline \multicolumn{4}{|l|}{ TNM stage } \\
\hline $\mathrm{I}+\mathrm{II}$ & 61 & $1.675 \pm 0.881$ & 0.0138 \\
\hline III + IV & 144 & $2.117 \pm 1.076$ & \\
\hline \multicolumn{4}{|l|}{ Differentiation } \\
\hline Well & 46 & $1.964 \pm 0.954$ & 0.8732 \\
\hline Moderate and low & 144 & $1.993 \pm 1.052$ & \\
\hline \multicolumn{4}{|l|}{ Lymph node metastases } \\
\hline Negative & 74 & $1.719 \pm 0.917$ & 0.0356 \\
\hline Positive & 131 & $2.136 \pm 1.077$ & \\
\hline \multicolumn{4}{|l|}{ Distant metastases } \\
\hline Negative & 155 & $1.867 \pm 1.005$ & 0.0004 \\
\hline Positive & 50 & $2.354 \pm 1.069$ & \\
\hline
\end{tabular}

reaction monitoring mass spectrometry to investigated the circulating concentration of haptoglobin subunits in 210 NSCLC patients, found serum levels of haptoglobin alpha and beta chains were obviously elevated in NSCLC compared with controls, could be a biomarker for the diagnosis of NSCLC, but lacking of prognostic data [6]. In this study, we detected serum haptoglobin levels in 205 NSCLC patients by commercially available human haptoglobin assay kit, using a fully automated analytical platform, Beckman Coulter AU5800. Our sample's size is adequate, and detect method is very convenient and suitable for the most of primary hospital, which is very important for the vast rural of China and other developing and undeveloped countries.

We verified whether serum haptoglobin levels associated with NSCLC lymph node metastasis or not. The area under the ROCs for the detection of NSCLC lymph node metastasis by serum haptoglobin was 0.609 (sensitivity of 0.672 , specificity of 0.554 ), suggested that haptoglobin might be associated with the progression and poor prognosis of NSCLC. More interesting, another acute-phase protein, C-reactive protein also was found elevated in NSCLC patients, and associated with disease progression and poor prognosis $[22,23]$. The precise molecular mechanism for the function of haptoglobin in cancer pathogenesis is still unclear, by now. We speculated that serum haptoglobin levels might be reflected seriousness of infection, which could be caused by tumor necrosis or metastasis. This might suggested that haptoglobin is a potential prognostic biomarker, even the molecular therapeutic target for NSCLC.

However, there were still several limitations in this study. First, this is a single-center study, a large multicenter 
studies will be needed to adjust the possible biases, to more precisely evaluate the value of serum haptoglobin as a biomarker of NSCLC. Second, the ratio of clinical stage was not ideal. Most of cases were in the advanced stages (III + IV, 70.2\%). Third, CA125, CEA, CYFRA21-1 and

A

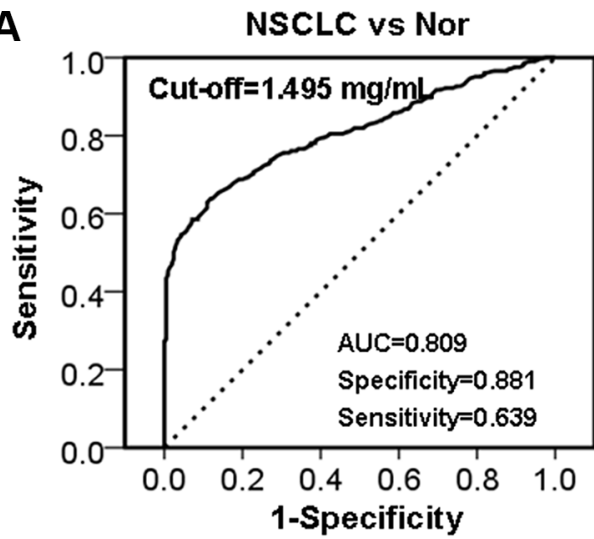

C

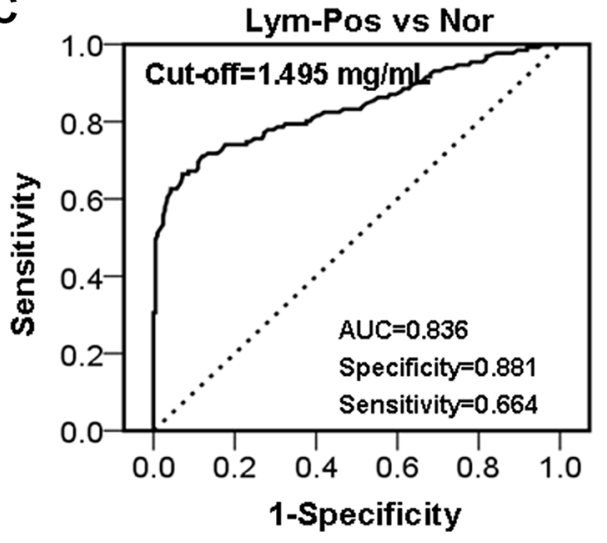

SCC had not been evaluated; we were not able to compare the diagnostic power of serum haptoglobin with existing biomarker in NSCLC. Last, the exact mechanism of haptoglobin for cancer's tumorigenesis and progression was not clear, by now.
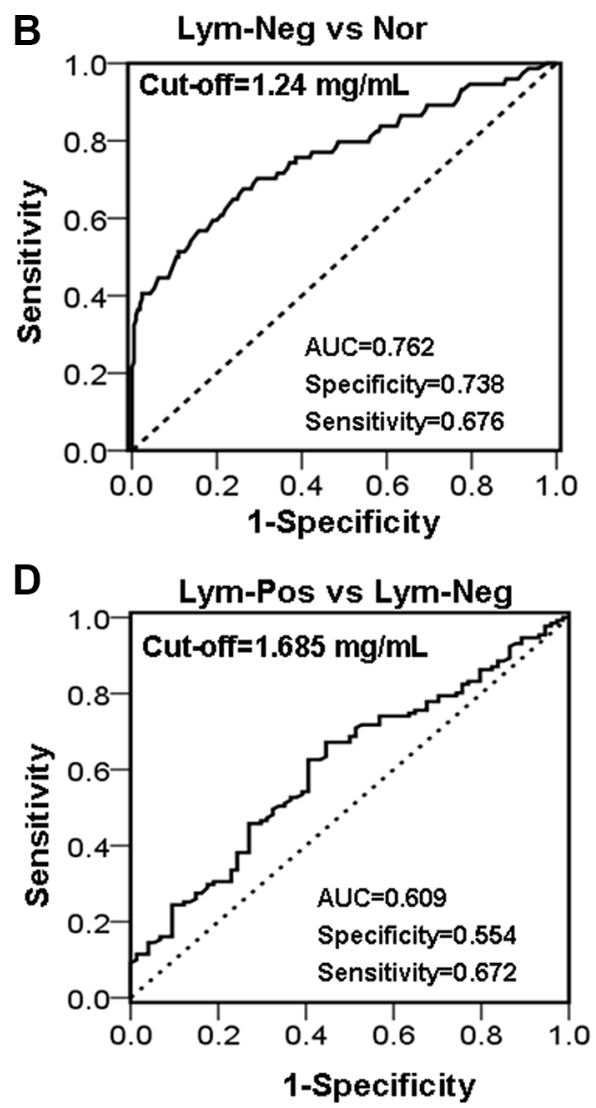

Figure 2: ROC analyses for serum haptoglobin to differentiate (A) NSCLC from normal healthy controls; (B) NSCLC patients with LymNeg from Nor; (C) NSCLC patients with Lym-Pos from Nor; (D) NSCLC patients with Lym-Pos from Lym-Neg. Nor, Normal healthy controls, Lym-Neg, Lymph node metastasis negative, Lym-Pos, Lymph node metastasis positive.

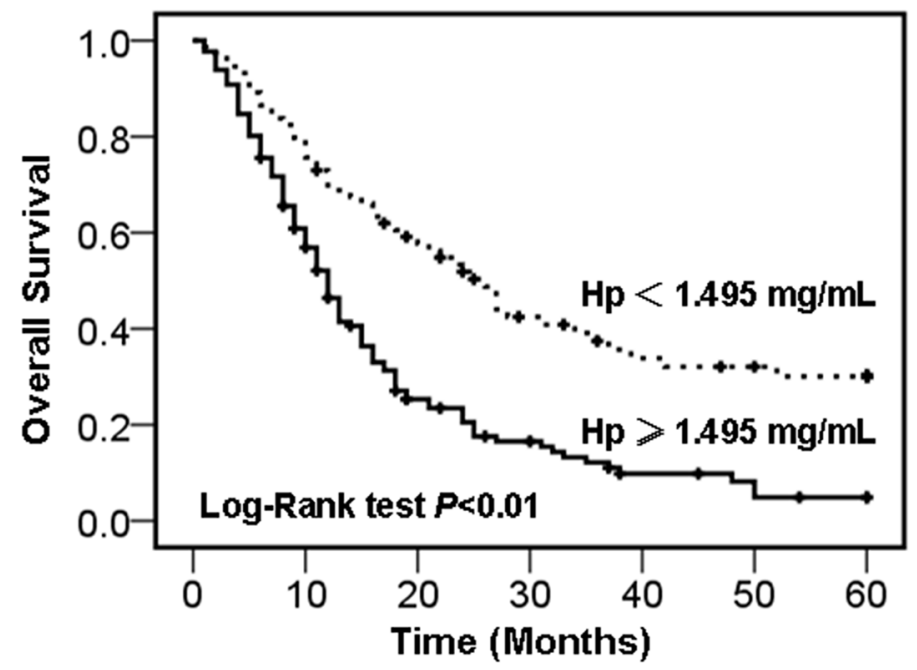

Figure 3: Kaplan-Meier survival curves of NSCLC patients. Overall survival rate of NSCLC patients with high- and low- serum haptoglobin levels group. 
Table 3: Univariate and multivariate Cox analysis of variables considered for overall survival rates of NSCLC patients

\begin{tabular}{lccccc}
\hline \multicolumn{1}{c}{ Variables } & Category & $\begin{array}{c}\text { Univariate } \\
\boldsymbol{P} \text { value }\end{array}$ & HR & $\begin{array}{c}\text { Multivariate } \\
\mathbf{9 5 \%} \text { CI }\end{array}$ & $\boldsymbol{P}$ value \\
\hline Age & $>60$ vs. $<60$ years & 0.45 & 1.07 & $0.52-2.18$ & 0.37 \\
Gender & male vs. female & 0.63 & 0.95 & $0.58-1.68$ & 0.26 \\
Smoking status & smoker vs. non-smoker & 0.32 & 1.18 & $0.34-2.13$ & 0.34 \\
TNM stage & III + IV vs. I + II & $<0.01$ & 3.38 & $1.83-8.63$ & $<0.01$ \\
Differentiation & well vs. moderate + low & 0.08 & 0.88 & $0.44-1.52$ & 0.33 \\
Lymph node metastases & positive vs. negative & $<0.01$ & 2.89 & $1.31-6.84$ & $<0.01$ \\
Distant metastases & positive vs. negative & $<0.01$ & 4.53 & $2.01-7.94$ & $<0.01$ \\
Serum haptoglobin levels & high vs. low & $<0.01$ & 2.45 & $1.47-4.49$ & 0.01 \\
\hline
\end{tabular}

Abbreviations: $\mathrm{HR}=$ hazards ratio; $\mathrm{CI}=$ confidence interval.

In summary, our results showed that serum haptoglobin levels significantly elevated in NSCLC patients compared with normal healthy controls, and there was a strong association between high serum haptoglobin levels and TNM stages, lymph node metastasis, and distant metastasis. Furthermore, NSCLC patients with higher serum haptoglobin levels had poorer prognosis, suggesting that serum haptoglobin may act as a useful clinical serological biomarkers in diagnosing, progression and prognostic evaluation in NSCLC. These results also suggested that haptoglobin might be a potential therapeutic target for the treatment of NSCLC. However, this hypothesis needed to be explored by further study.

\section{MATERIALS AND METHODS}

\section{Patient population and specimens}

Two hundred and five NSCLC patients of Han Chinese, including one hundred and sixteen patients with adenocarcinoma, seventy-six patients with squamous cell carcinoma, and thirteen patients with other subtypes of NSCLC diagnosed and treated in the First Affiliated Hospital of Sun Yat-sen University in the southern of China, from January 2010 to January 2012 were enrolled in this study. All NSCLC patients' diagnoses were confirmed independently by two pathologists, who reviewed pathological slides of tissue from biopsy or resected specimens. Patients who suffered hyperlipidemia, infection, liver disease, previous malignancy, received antibiotic treatment, or received adjuvant therapy before surgery were excluded from the study. All NSCLC patients received standard treatment with routine therapy, chemotherapy, and radiotherapy after the surgery operation according to NCCN guideline for NSCLC. All patients' histopathological classification was determined according to the WHO criteria, and staged classification was defined according to the 7th edition of UICC TNM staging system $[24,25]$. Two hundred and ten normal healthy controls were recruited from healthy unrelated subjects who performed routine yearly health check-up at the Physical Health Examination Centre of our medical center whose age and gender matched subjects who did not have any family history cancer were recruited in this study.

This study was approved by the Human Research Ethics Committee of the First Affiliated Hospital of Sun Yat-sen University according to the guidelines of Helsinki conventions. All participants were informed the information of the purpose of the study and the experimental procedures, and given written informed consent and applied a standardized questionnaire to gather demographic and personal information at the first visit. All subjects with NSCLC were followed up at intervals of one to two months until December 2015, and follow up time ranged from one to sixty months.

\section{Data collection and laboratory tests}

The patients' clinicopathological variables were collected, including age, sex, treatment, histopathological findings, stage, and medication history. Prior to treatment, blood were collected from each subject in the morning between $7 \mathrm{AM}$ and $10 \mathrm{AM}$ for serum tests. To minimize the influence of diet on detecting serum haptoglobin levels, each subjects overnight fast of at least 8 hours before blood collected. Collected blood samples were allowed to clot and serum were separated by centrifugation at 3,000 rpm for $10 \mathrm{~min}$ at $4^{\circ} \mathrm{C}$, and then determined in the clinical laboratory of the First Affiliated Hospital of Sun Yat-sen University, immediately. Blood fat and hemolytic sample was excluded from the study. Serum haptoglobin levels were determined by a commercially available human haptoglobin assay kit by immunoturbidimetry method (Tridelta PHASE Haptoglobin Assay, County Kildare, Ireland), using a fully automated analytical platform (Beckman Coulter AU5800, Beckman Coulter, Inc., USA). All tests were detected according to the manufacturer's protocols. 


\section{Statistical analyses}

All data statistical analyses were performed using Graphpad Prism version 5.0 (GraphPad Software Inc., San Diego, CA, USA) and SPSS version 13.0 (SPSS Inc., Chicago, IL, USA). All variables under normal distribution were shown as the mean \pm standard deviation (SD). The differences between groups were determined by Mann-Whitney $U$ test and Kruskal-Wallis test. The association between haptoglobin expression levels and various clinicopathological variables in NSCLC were evaluated by using Mann-Whitney $U$ test or the Wilcoxonmatched test, and Pearson chi-square test or Fisher's exact test was tested for categorical values. The statistical power was calculated by using the Power and Precision software version 4 (Biostat, NJ, USA). Receiver operating characteristics (ROC) analysis was plotted to determine the sensitivity and specificity of serum haptoglobin levels to discriminate between NSCLC and healthy controls or stratify patients at a high risk of metastasis. The diagnostic power of serum haptoglobin was assessed by sensitivity, specificity, and area under ROC curve (AUC). The cutoff value was determined by the score closest the value under both peak of sensitivity and specificity. Survival rates and curves were determined by the Kaplan-Meier method, and the comparison of differences of survival was evaluated by using the log-rank test. COX regression analysis was used for univariate and multivariate analysis of correlation between clinicopathological variables and overall survival. In all cases, $P$ values less than 0.05 were considered statistically significant, and all statistical tests were two-sided.

\section{ACKNOWLEDGMENTS AND FUNDING}

The work was supported by grants from the Specialized Research Fund for the Doctoral Program of Higher Education of China (Grant No. 20130171120069 to LSL), and the Science and Technology Department of Guangdong Province (Grant No. 2014A020212477 to LSL, 2014A020212720 to JML), and the Natural Science Foundation of Guangdong Province, China (Grant No. 2015A030313035 to LSL), and the Science and Technology Department of Guangzhou City, China (Grant No. 201400000004-2 to ML). The funder had no role in study design, data collection and analysis, decision to publish, or preparation of the manuscript.

\section{CONFLICTS OF INTEREST}

We declare that we have no conflicts of interest.

\section{REFERENCES}

1. Chen W, Zheng R, Baade PD, Zhang S, Zeng H, Bray F, Jemal A, Yu XQ, He J. Cancer statistics in China, 2015. CA Cancer J Clin. 2016; 66:115-132.
2. Herbst RS, Heymach JV, Lippman SM. Lung cancer. The New England journal of medicine. 2008; 359:1367-1380.

3. Gumireddy K, Li A, Chang DH, Liu Q, Kossenkov AV, Yan J, Korst RJ, Nam BT, Xu H, Zhang L, Ganepola GA, Showe LC, Huang Q. AKAP4 is a circulating biomarker for non-small cell lung cancer. Oncotarget. 2015; 6:17637-17647. doi: 10.18632/oncotarget.3946.

4. National Lung Screening Trial Research T, Aberle DR, Adams AM, Berg CD, Black WC, Clapp JD, Fagerstrom RM, Gareen IF, Gatsonis C, Marcus PM, Sicks JD. Reduced lung-cancer mortality with low-dose computed tomographic screening. The New England journal of medicine. 2011; 365:395-409.

5. Kulpa J, Wojcik E, Reinfuss M, Kolodziejski L. Carcinoembryonic antigen, squamous cell carcinoma antigen, CYFRA 21-1, and neuron-specific enolase in squamous cell lung cancer patients. Clinical chemistry. 2002; 48:1931-1937.

6. Park J, Yang JS, Jung G, Woo HI, Park HD, Kim JW, Huh W, Ko JW, Kim H, Cho JY, Lee SY. Subunit-specific mass spectrometry method identifies haptoglobin subunit alpha as a diagnostic marker in non-small cell lung cancer. Journal of proteomics. 2013; 94:302-310.

7. Tubbs KA, Kiernan UA, Niederkofler EE, Nedelkov D, Bieber AL, Nelson RW. High-throughput MS-based protein phenotyping: application to haptoglobin. Proteomics. 2005; 5:5002-5007.

8. Quaye IK. Haptoglobin, inflammation and disease. Transactions of the Royal Society of Tropical Medicine and Hygiene. 2008; 102:735-742.

9. Oh MK, Park HJ, Lee JH, Bae HM, Kim IS. Single chain precursor prohaptoglobin promotes angiogenesis by upregulating expression of vascular endothelial growth factor (VEGF) and VEGF receptor2. FEBS letters. 2015; 589:1009-1017.

10. Heegaard PM, Klausen J, Nielsen JP, Gonzalez-Ramon N, Pineiro M, Lampreave F, Alava MA. The porcine acute phase response to infection with Actinobacillus pleuropneumoniae. Haptoglobin, C-reactive protein, major acute phase protein and serum amyloid A protein are sensitive indicators of infection. Comparative biochemistry and physiology Part B, Biochemistry \& molecular biology. 1998; 119:365-373.

11. Hoagland LFt, Campa MJ, Gottlin EB, Herndon JE, 2nd and Patz EF, Jr. Haptoglobin and posttranslational glycan-modified derivatives as serum biomarkers for the diagnosis of nonsmall cell lung cancer. Cancer. 2007; 110: 2260-2268.

12. Lee CC, Ho HC, Chien SH, Hsiao SH, Hung SK, Huang TT, Yu CC, Chang SM, Huang HH, Su YC. Association of acute phase protein-haptoglobin, and epithelial-mesenchymal transition in buccal cancer: a preliminary report. Clinical chemistry and laboratory medicine. 2013; 51:429-437.

13. Wang B, He YJ, Tian YX, Yang RN, Zhu YR, Qiu H. Clinical utility of haptoglobin in combination with CEA, 
NSE, CYFRA21-1 for diagnosis of lung cancer. Asian Pacific journal of cancer prevention. 2014; 15:9611-9614.

14. Sun L, Hu S, Yu L, Guo C, Sun L, Yang Z, Qi J, Ran Y. Serum haptoglobin as a novel molecular biomarker predicting colorectal cancer hepatic metastasis. International journal of cancer. 2016; 138:2724-2731.

15. Stieber P, Holdenrieder S. Lung cancer biomarkers - Where we are and what we need. Cancer biomarkers. 2010; 6: 221-224.

16. Molina R, Holdenrieder S, Auge JM, Schalhorn A, Hatz R, Stieber P. Diagnostic relevance of circulating biomarkers in patients with lung cancer. Cancer biomarkers. 2010; 6: 163-178.

17. Korbakis D, Dimitromanolakis A, Prassas I, Davis GJ, Barber E, Reckamp KL, Blasutig I, Diamandis EP. Serum LAMC2 enhances the prognostic value of a multiparametric panel in non-small cell lung cancer. British journal of cancer. 2015; 113:484-491.

18. Gebauer F, Gelis S, Zander H, Karstens KF, WoltersEisfeld G, Izbicki JR, Bockhorn M, Tachezy M. Tenascin-C serum levels and its prognostic power in non-small cell lung cancer. Oncotarget. 2016; doi: 10.18632/oncotarget.7976.

19. Barak V, Holdenrieder S, Nisman B, Stieber P. Relevance of circulating biomarkers for the therapy monitoring and follow-up investigations in patients with non-small cell lung cancer. Cancer biomarkers. 2010; 6:191-196.

20. Yang D, Zhou J, Zeng T, Yang Z, Wang X, Hu J, Song Y, Chen L, Peer D, Wang X, Bai C. Serum chemokine network correlates with chemotherapy in non-small cell lung cancer. Cancer letters. 2015; 365:57-67.
21. Wikoff WR, Hanash S, DeFelice B, Miyamoto S, Barnett M, Zhao Y, Goodman G, Feng Z, Gandara D, Fiehn O, Taguchi A. Diacetylspermine Is a Novel Prediagnostic Serum Biomarker for Non-Small-Cell Lung Cancer and Has Additive Performance With Pro-Surfactant Protein B. Journal of clinical oncology. 2015; 33:3880-3886.

22. Lee JG, Cho BC, Bae MK, Lee CY, Park IK, Kim DJ, Ahn SV, Chung KY. Preoperative C-reactive protein levels are associated with tumor size and lymphovascular invasion in resected non-small cell lung cancer. Lung cancer. 2009; 63:106-110.

23. Fiala O, Pesek M, Finek J, Topolcan O, Racek J, Minarik M, Benesova L, Bortlicek Z, Poprach A, Buchler T. High serum level of C-reactive protein is associated with worse outcome of patients with advanced-stage NSCLC treated with erlotinib. Tumour biology. 2015; 36:9215-9222.

24. Goldstraw P, Crowley J, Chansky K, Giroux DJ, Groome PA, Rami-Porta R, Postmus PE, Rusch V, Sobin L, International Association for the Study of Lung Cancer International Staging C, Participating I. The IASLC Lung Cancer Staging Project: proposals for the revision of the TNM stage groupings in the forthcoming (seventh) edition of the TNM Classification of malignant tumours. Journal of thoracic oncology. 2007; 2:706-714.

25. Brambilla E, Travis WD, Colby TV, Corrin B, Shimosato Y. The new World Health Organization classification of lung tumours. Eur Respir J. 2001; 18:1059-1068. 